\title{
L'emodialisi domiciliare: l'esperienza di un piccolo Centro
}

\author{
Pietro Bajardi
}

\author{
Dipartimento delle Malattie del Rene e delle Vie Urinarie \\ Unità Operativa di Nefrologia e Dialisi, Regione Piemonte, ASL 12, Biella
}

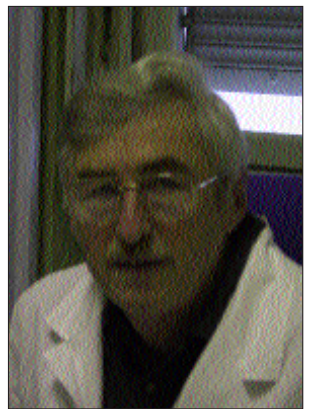

【 evoluzione dell'utilizzo dell'emodialisi d o miciliare (HDD) è singolarmente sovrapponibile nelle diverse aree geografiche come risulta dai diversi registri (USRDS, Registro Italiano ecc.), con una prevalenza di pazienti trattati scesa nel 1999 fino all' $1.7 \%$ negli USA e all' $1 \%$ in Italia. Anche l'esempio di un piccolo Centro quale il nostro (28 pazienti inviati in dialisi domiciliare complessivamente) ricalca tale andamento (Fig. 1). Poiché l'attività del nostro Centro è iniziata alla fine degli anni '70, manca nel grafico la fase caratteristica del precedente decennio di rapida espansione della HDD legata alla carenza di posti rene nei Centri e alla scelta obbligata: "go home or die". Ma l'inizio dell'attività, in una fase in cui la CAPD muoveva solo i primi passi e riconosceva un tasso di una peritonite ogni 3 mesi/paziente e i Centri ad assistenza limitata (CAL) non erano ancora una soluzione comune, vedeva la HDD raggiungere rapidamente il $20 \%$ della nostra po- polazione dialitica. Gli anni successivi, con i significativi miglioramenti tecnici e quindi col rapido affermarsi della dialisi peritoneale (PD), dapprima con la CAPD e in seguito con la APD notturna, e poi con l'apertura di due CAL, la HDD è progressivamente discesa a rappresentare una metodica marginale che riguarda ora solo il $4 \%$ della popolazione dialitica $(6 / 135$ pazienti).

Quando una tecnica viene progressivamente abbandonata col sopraggiungere di altre è ovvia conseguenza ritenere che essa presenti sostanziali svantaggi rispetto alle tecniche nuove.

E questo il caso della dialisi domiciliare?

I migliori risultati della HDD rispetto alla dialisi in Centro, sono stati definitivamente accertati dall'indagine di Woods (1) con una analisi retrospettiva "intent to treat": su una popolazione di 4892 pazienti del registro USRDS, il rischio relativo di morte era per $\mathrm{i}$ pazienti in HDD, rispetto ai pazienti trattati in Centro, di $0.37 \mathrm{e}$ saliva a 0.58 , con differenza tuttora significativa, se aggiustato per età, sesso, razza, diabete e fattori comorbidi, derivando verosimilmente tale dato più che da differenze di prescrizione o di tecniche dialitiche in sé da una applicazione di esse più consapevole e attenta. Questo dato, già peraltro segnalato fin dagli anni '70 (2), ci ha spinto a proporre, qualche volta con insistenza, la HDD ove possibile

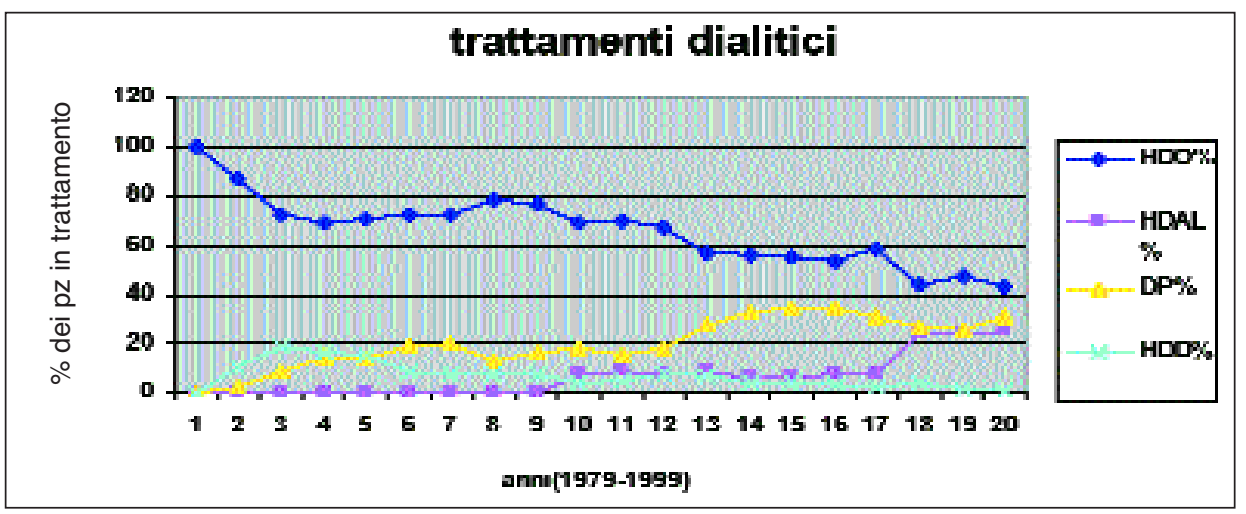

Fig. 1 
ai pazienti di nuova immissione. Tuttavia obiettive condizioni pratiche ne limitavano lo sviluppo. La HDD richiede infatti alcune condizioni di base:

a) assenza di controindicazioni cliniche;

b) domicilio adatto possibilmente con un locale attrezzato (nella nostra esperienza tuttavia 7 pazienti, pari al $25 \%$ della popolazione eseguivano le sedute nella camera da letto);

c) buon accesso vascolare;

d) capacità di apprendimento sufficienti per utilizzare procedure relativamente complesse e buona adesione alle prescrizioni;

e) presenza di un partner stabile e affidabile (nella nostra casistica la moglie in 22 casi (78\%), il marito in due casi, in due la madre e in un caso il padre e il figlio).

$\mathrm{La} \mathrm{PD}$, viceversa, può esser praticata anche in assenza delle condizioni di cui ai punti c) ed e) e con condizioni molto più facilitate rispetto ai punti b) e d), mentre rispetto al punto a) le condizioni richieste sono meno restrittive, essendo praticabile, anzi a volte indicata, anche in pazienti ad alto rischio. Inoltre rispetto alla HDD la PD presenta alcuni vantaggi organizzativi (semplicità delle manovre, training rapido e rapido invio a domicilio, costi contenuti ecc.).

Dal punto di vista strettamente clinico la HDD è stata dimostrata superiore anche alla PD rispetto a mortalità e morbidità in uno studio che confrontava due gruppi di 139 pazienti controllati per sesso ed età. I fattori di rischio cardiaco tuttavia non erano omogeneamente distribuiti ed erano significativamente più bassi nel gruppo in HDD (3).

Peraltro anche dal punto di vista strettamente clinico la PD presenta un vantaggio decisivo. Il mantenimento di un filtrato glomerulare residuo è un requisito di grande importanza per la depurazione globale, specialmente per molecole di maggiori dimensioni o legate alle proteine, oltre a essere di grande aiuto per il mantenimento dell'omeostasi idroelettrolitica (4). Dopo molte precedenti segnalazioni, la maggior capacità della PD a mante- nere la funzione renale residua è stata recentemente definitivamente dimostrata (5). Con l'attuale indicazione a iniziare la terapia dialitica con un $\mathrm{Kt} / \mathrm{V}$ urea $=2(6)$, vale a dire per una persona di $70 \mathrm{~kg}$, con una clearance dell'urea di circa 7-8 $\mathrm{ml} / \mathrm{min}$, cui corrisponde in genere una creatinina clearance tra 10 e $15 \mathrm{ml} / \mathrm{min}$, cioè con una funzione residua significativa, questa caratteristica assume una grande importanza.

La PD si propone come il trattamento di scelta per quei pazienti che si collocano con un FG tra i 10 e $5 \mathrm{ml} / \mathrm{min}$, così come la terapia conservativa è il trattamento ideale per i pazienti con un filtrato superiore a questi livelli, mentre con FG inferiori, vi può essere, per esigenze depurative, un'indicazione preferenziale per la HD. È nostra opinione che, con l'esclusione di coloro che hanno specifiche controindicazioni, tutti i pazienti uremici, in questa fase, dovrebbero essere sottoposti a un periodo di trattamento peritoneale. Per tale motivo, nella nostra esperienza la DP è stato il maggior fattore limitante l'estensione della HDD. Se escludiamo il periodo dei primi anni ' 80 , quando la PD era ancora un trattamento poco consolidato, i pazienti da noi trattati con HDD erano stati esclusi dalla PD o per controindicazioni a tale trattamento, $\mathrm{o}$ per problemi connessi all'organizzazione della giornata lavorativa, $\mathrm{o}$, più frequentemente, specie nei giovani, per problemi psicologici relativi all'applicazione del catetere di Tenkoff. Per tali pazienti in effetti faceva premio sul dato clinico la miglior qualità di vita documentata per la HDD (7). La scelta dei nostri pazienti è stata determinata in 14 casi $(50 \%)$ da esigenze connesse all'attività lavorativa, mentre nei restanti prevalevano le esigenze di una migliore organizzazione del tempo libero o il desiderio di svolgere il trattamento nell'ambiente familiare.

La riduzione dei costi di trattamento è riconosciuta come una delle principali motivazioni a estendere il trattamento con HDD, risultando essa nelle diverse analisi su costi e tariffe, concordi nei differenti Paesi, seconda solo alla CAPD (8). È stata posta la questione se in un piccolo Centro con pochi pazienti in trattamento la HDD mantenga il suo vantaggio economico ed è stato proposto di convogliare solo in pochi Centri selezionati la gestione della dialisi a domicilio (9).

Tale soluzione può esser proponibile in una grande città con più Centri dialisi, ma è difficilmente praticabile in realtà decentrate. Peraltro i costi di gestione possono essere ridotti evitando di mantenere una équipe dedicata: la sorveglianza medica telefonica durante il trattamento ora non richiede più la presenza fisica in ospedale e può essere risolta con una reperibilità più rigorosa, mentre l'équipe medico-infermieristica nella nostra situazione è stata unificata con quella della dialisi peritoneale con risultati soddisfacenti anche per il personale. Il vantaggio economico resta soprattutto legato alla permanenza del paziente in trattamento che deve esser tale da ammortizzare i maggiori costi iniziali per l'addestramento. Due studi diversi hanno valutato il cosiddetto "pay back time", cioè il periodo necessario per tale ammortamento in 14.4 e rispettivamente 14.2 mesi ( 9 , 10) Tutti i nostri pazienti tranne uno, morto dopo 6 mesi, tra i 22 usciti dal trattamento, hanno superato il "pay back time". La durata media della metodica è stata di 66.36 mesi (min. 6, max. 236 mesi).

Il drop-out a differenza che in PD dove sono determinanti le complicazioni della metodica, nel nostro gruppo di HDD è stato determinato in 13 casi (59\%) da trapianto, in 4 casi $(18 \%)$ da sopravvenuta inidoneità clinica, in 2 casi (9\%) da problemi sociofamiliari e in $3(13 \%)$ da morte. E ovvio che, almeno nella nostra situazione, lo sviluppo della HDD è inverso a quello dei programmi di trapianto. Questo potrà però non essere più vero $\mathrm{se}$, con le nuove tecniche che si prospettano oggi (HDD domiciliare notturna, breve quotidiana) la HDD si orienterà a trattamenti anche per situazioni cliniche più compromesse, e quindi anche per una popolazione di pazienti che non abbia il trapianto come prospettiva immediata. 


\section{BIBLIOGRAFIA}

riod. J Am Med Assoc 1981; 246:

230-2.

1. Woods JD, Port FK, Stannard D, Blagg CR, Held PJ. Comparison of mortality with home hemodialysis and center hemodialysis: A national study. Kidney Int 1996; 49: 1464-70.

2. Vercellone A, et al. Tavola rotonda: la dialisi domiciliare - Attualità Nefrologiche e Dialitiche 1976.

3. Grant AG, et al. Dialysis at home in West Scotland: a comparison of hemodialysis and CAPD in age and sex matched controls. Perit Dial Int 1992; 12: 365-8.

4. Dhont A, et al. The removal of uremic toxins. Kidney Int 2000; 58 (suppl): S47-59.

5. Misra M, Vonesh E, Van Stone JC, Moore HL, Prowant B, Nolph KD. Effect of cause and time of dropout on the residual GFR: a comparative analiysis of the decline of GFR in dialysis. Kidney Int 2001; 59: 754-63.

6. K/DOQI. Clinical Practice Guidelines for peritoneal dialysis adequacy.

7. Oberley ET, et al. Home hemodialysis: survival, quality of life, and rehabilitation. Adv Ren Replace Ther 1996; 3: 147-53.

8. De Vecchi AF, Dratwa M, Wiedemann ME. Healthcare system and ESRD therapy-an international review: cost and reimbursment/funding of ESRD therapies. Nephrol Dial Transpl 1999; 14 (suppl): S31-41.

9. Mackenzie PJ and Murphy TH. Home haemodialysis in the 1990 s. Nephrol Dial Transpl 1998; 13: 1994-8.

10. Delano BG, Feinroth MV, Feinroth M, Friedman EA. Home and medical centre hemodialysis. Dollar comparison and pay back pe- 